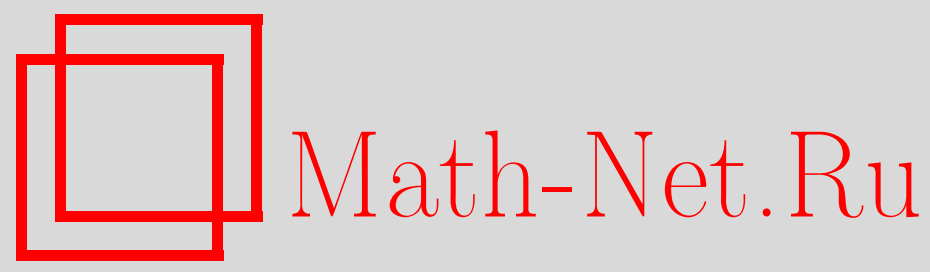

Ю. Ф. Коробейник, О связи между максимумом модуля и тейлоровскими коэффициентами целых функций многих комплексных переменных, Матем. заметки, 1997, том 62, выпуск 2, 238-258

DOI: https://doi.org/10.4213/mzm1608

Использование Общероссийского математического портала Math-Net.Ru подразумевает, что вы прочитали и согласны с пользовательским соглашением http://www . mathnet.ru/rus/agreement

Параметры загрузки:

IP : 54.205 .225 .156

26 апреля 2023 г., 16:29:21

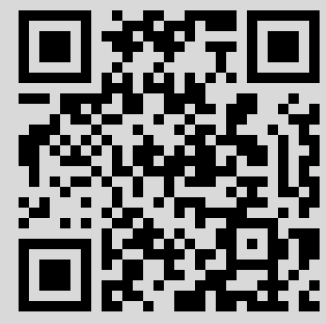




\title{
О СВЯЗИ МЕЖДУ МАКСИМУМОМ МОДУЛЯ \\ И ТЕЙЛОРОВСКИМИ КОЭФФИЦИЕНТАМИ \\ ЦЕЛЫХ ФУНКЦИЙ МНОГИХ КОМПЛЕКСНЫХ ПЕРЕМЕННЫХ
}

\author{
Ю. Ф. Коробейник
}

В работе приводятся результаты о связи между ростом максимума модуля и убыванием тейлоровских коэффициентов целых функций многих переменных. Результаты получены двумя различными способами, первый из которых в одномерной ситуации предложен ранее В.А. Осколковьм, а второй основан на использовании функций, сопряженных по Лежандру-Юнгу-Фенхелю с весовыми. Основное внимание уделяется характеризации роста целых функций по совокупности переменных; однако, некоторые резултаты получены и в случае, когда допускается различный рост по разным переменным.

Библиографиия: 8 названий.

Связь между характером возрастания максимума модуля целой функции одного и нескольких переменных и убьвания модулей ее тейлоровских коэффициентов исследовалась многими математиками, начиная с Адамара, которьй в конце XIX века ввел понятия порядка и типа целой функции одного комплексного переменного и выразил их через модули тейлоровских коэффициентов. При этом целая функция конечного ненулевого порядка может иметь нулевой или бесконечный (максимальньй) тип. В 20-х годах нашего века Валирон ввел понятие обобщенного порядка $\rho(r) \rightarrow \rho>0[1, \S 12]$ и показал, что для любой целой функции $f(z)$ ненулевого конечного порядка $\rho$ можно построить уточненный порядок $\rho(r) \rightarrow \rho$ такой, что $f$ имеет нормальньй (т.е. конечный и ненулевой) тип $\sigma$ относительно порядка $\rho(r)$ :

$$
\sigma=\varlimsup_{r \rightarrow \infty} r^{-\rho(r)} \ln M_{f}(r)
$$

где $M_{f}(r)=\max \{|f(z)|:|z|=r\}$. При этом, как показал Валирон, тип целой функции порядка $\rho \in(0,+\infty)$ относительно любого уточненного порядка $\rho(r) \rightarrow \rho$ также выражается с помошью довольно простой формулы через модули ее тейлоровских коэффицциентов.

В последнее время весьма обшие результаты по построению шкалы сравнения целых функций были получены Осколковым [2], [3]. В частности, он показал, что в некотором

Работа выполнена при финансовой поддержке Российского фонда фундаментальных исследований, грант № 93-011-242. 
классе $\bar{I}$ вешественнозначных функций, определенных на $[1, \infty)$, можно ввести $\alpha-m u n$ целой функции

$$
f(z)=\sum_{n=0}^{\infty} f_{n} z^{n}
$$

по правилу

$$
\sigma_{\alpha}(f)=\varlimsup_{r \rightarrow \infty}\left(\ln M_{f}(r) / \int_{1}^{r} \alpha(t) d \ln t\right)
$$

и выразить его через тейлоровские коэффициенты $f_{n}$. При этом для произвольной целой функции $f$ найдется функция $\alpha_{0}$ из класса $\bar{I}$, для которой $0<\sigma_{\alpha_{0}}(f)<+\infty[2]$, [3].

Осколков также показал, что для любой целой функции $f$ требуемую весовую функцию $\alpha_{0}\left(\right.$ с $\left.\sigma_{\alpha_{0}}(f)=1\right)$ можно выбрать в классе $\widehat{I}$, более узком, чем первоначальньй класс $\bar{I}$ (точное описание классов $\bar{I}$ и $\widehat{I}$, введенных в [2], [3], приводится ниже).

В связи с работами [2], [3] возник вопрос о справедливости аналогичных результатов для других функциональных рядов: одномерных и многомерных рядов Дирихле, Тейлора-Дирихле и т.д. Пожалуй, в таком исследовании естественно начать с многомерных степенных рядов, т.е. с целых функций нескольких комплексных переменньх. Именно характеризация роста целых в $\mathbb{C}^{m}, m \geqslant 1$, функций и является основным моментом в данной статье. Вначале, используя методы, развитые в [2], [3] для одномерного случая, получаются аналоги одномерных результатов по описанию роста по совокупности переменных целых в $\mathbb{C}^{m}$ функций. При этом некоторые из полученных в этом направлении результатов являются новыми и в случае $m=1$.

Далее в работе изучается случай, когда целая функция может иметь различньй рост по разным переменным. Именно, по аналогии с сопряженными типами при данной системе порядков $\left(\rho_{1}, \ldots, \rho_{m}\right)$, введенных Баумгартнером еще в 1914 году, в работе даются определения сопряженных $\alpha$-типов (по максимуму модуля функции $f$ и по ее тейлоровским коэффициентам) и показывается, что соответствующие две гиперповерхности сопряженных типов (по модулю функции и по ее тейлоровским коэффищиентам) совпадают. Дается законченное описание гиперповерхности сопряженных $\alpha$-типов по модулю функции (указать столь же полное описание второй гиперповерхности удается пока лишь при дополнительных, довольно жестких, ограничениях на функцию $\alpha(x))$.

В последней части статьи для описания исследуемой связи применяется другой метод, основанный на использовании функций, сопряженных к весовьм в смысле Лежандра-Юнга-Фенхеля. Такой подход позволил охарактеризовать порядок убьвания коэффициентов целых в $\mathbb{C}^{m}$ функций, мажоранты роста которых имеют довольно сложную (например, двучленную) асимптотику.

1. Основные результаты для описания роста по совокупности переменных. Пусть $m \geqslant 1$ и $A\left(\mathbb{C}^{m}\right)$ - пространство всех целых функций

$$
f(z)=\sum_{\|n\|=0}^{\infty} f_{n} z^{n}
$$

где $n=\left(n_{1}, \ldots, n_{m}\right) \in \mathbb{Z}_{+}^{m},\|n\|=n_{1}+n_{2}+\cdots+n_{m}, z^{n}=z_{1}^{n_{1}} \cdots z_{m}^{n_{m}}$.

Пусть, далее, $I$ - множество вещественнозначных непрерьвно дифференцируемых на $[1, \infty)$ функций $\alpha(x)$ таких, что

1) $\alpha(1)=0$; 
2) $\alpha^{\prime}(x)>0 \forall x>1$

3) $\lim _{x \rightarrow \infty} \alpha(x)=+\infty$;

$\bar{I}$ - множество всех функций $\alpha$ из $I$, для которых

4) $\lim _{x \rightarrow \infty} \frac{x \alpha^{\prime}(x)}{\alpha^{2}(x)}=0$.

Обозначим символом $\beta(r)$ функцию, обратную к $\alpha(x) \in I$. Для любой $f$ из $A\left(\mathbb{C}^{m}\right)$ и для произвольной зафиксированной полной ограниченной кратнокруговой области $\mathscr{G} \subset \mathbb{C}^{m}$ с центром в начале координат (см. [4, с. 10$]$ или $[5$, с. 38$\left.]\right)$ положим

$$
\begin{gathered}
d_{n}(\mathscr{G})=\max \left\{\left|z_{1}\right|^{n_{1}} \cdots\left|z_{m}\right|^{n_{m}}: z \in \overline{\mathscr{G}}\right\}, \quad n \in \mathbb{Z}_{+}^{m}, \\
b_{n}=\left|f_{n}\right| d_{n}(\mathscr{G}), \quad M(R ; \mathscr{G}, f)=\max \left\{|f(z)|: z \in R^{\overline{\mathscr{G}}}\right\}, \\
R^{\overline{\mathscr{G}}}=\{w=R v: v \in \overline{\mathscr{G}}\},
\end{gathered}
$$

как обычно, $\overline{\mathscr{G}}$ - замькание области $\mathscr{G}$.

ПРЕДЛОЖЕНИЕ 1. Пусть $\alpha \in I, f \in A\left(\mathbb{C}^{m}\right)$ и существуют $\sigma>0, r_{0}<\infty$ такие, чmo

$$
\ln M(r ; \mathscr{G}, f) \leqslant \sigma \int_{1}^{r} \frac{\alpha(t)}{t} d t \quad \forall r \geqslant r_{0} .
$$

Тогда найдется $n_{0}<\infty$ такое, что

$$
\ln b_{n}<-\int_{0}^{\|n\|} \ln \beta\left(\frac{t}{\sigma}\right) d t \quad \forall n \in \mathbb{Z}_{+}^{m}, \quad\|n\| \geqslant n_{0} .
$$

ДокАЗАТЕЛЬСТво. Согласно [4, с. 139] $b_{n} r\|n\| \leqslant M(r ; \mathscr{G}, f) \forall r>0, \forall n \in \mathbb{Z}_{+}^{m}$. Из (1) находим

$$
\ln b_{n} \leqslant \sigma \int_{1}^{r} \frac{\alpha(t)}{t} d t-\|n\| \ln r \quad \forall r \geqslant r_{0} .
$$

Выберем $n_{0}$ так, чтобы $n_{0} / \sigma>r_{0}$. Тогда, полагая $r=\beta(\|n\| / \sigma)$, получим при $\|n\| \geqslant n_{0}$

$$
\ln b_{n} \leqslant \sigma \int_{0}^{\|n\| / \sigma} \frac{t \beta^{\prime}(t)}{\beta(t)} d t-\|n\| \ln \beta\left(\frac{\|n\|}{\sigma}\right)=-\int_{0}^{\|n\|} \ln \beta\left(\frac{t}{\sigma}\right) d t .
$$

ПРЕДЛОЖЕНИЕ 2. Пусть $\alpha \in \bar{I}, f \in A\left(\mathbb{C}^{m}\right)$ и существуют $\sigma>0, n_{0}<\infty, d<\infty$ такие, что

$$
\ln b_{n} \leqslant-\int_{0}^{\|n\|} \ln \beta\left(\frac{t}{\sigma}\right) d t+d \ln \|n\| \quad \forall n \in \mathbb{Z}_{+}^{m}, \quad\|n\| \geqslant n_{0},
$$

Тогда для любого $\varepsilon>0$ найдется $r_{\varepsilon}<\infty$ такое, что

$$
\ln M(r ; \mathscr{G}, f) \leqslant(\sigma+\varepsilon) \int_{1}^{r} \frac{\alpha(t)}{t} d t \quad \forall r \geqslant r_{\varepsilon} .
$$


ДокАЗАТЕЛЬСТво. Из (2) следует, что найдется $d_{1}<\infty$ такое, что

$$
\ln b_{n} \leqslant d_{1}-\int_{0}^{\|n\|} \ln \beta\left(\frac{t}{\sigma}\right) d t+d \ln \|n\| \quad \forall n \in \mathbb{Z}_{+}^{m} .
$$

Для всех $r>0$ имеем

$$
\begin{gathered}
M(r ; \mathscr{G}, f) \leqslant \sum_{\|n\|=0}^{\infty} b_{n} r^{\|n\|} \leqslant M \sum_{\|n\|=0}^{\infty}(\|n\|+1)^{m-1+d} r^{\|n\|} \exp \left(-\int_{0}^{\|n\|} \ln \beta\left(\frac{t}{\sigma}\right) d t\right) \\
=M \exp \left(\sigma+\frac{\varepsilon}{2}\right) \int_{1}^{r} \frac{\alpha(t)}{t} d t \sum_{\|n\|=0}^{\infty}(\|n\|+1)^{m-1+d} B_{\|n\|} \exp \left(-\int_{0}^{\|n\|} \ln \beta\left(\frac{t}{\sigma}\right) d t\right), \\
\text { где } B_{k}=\max \left\{r^{k} \exp \left(-\left(\sigma+\frac{\varepsilon}{2}\right) \int_{1}^{r} \frac{\alpha(t)}{t} d t\right): r \geqslant 0\right\}, \quad k=0,1, \ldots
\end{gathered}
$$

Величина $B_{k}$ определяется с помощью элементарных выкладок (см. [2, с. 133]) и ее значение равно

$$
\exp \int_{0}^{k} \ln \beta\left(\frac{2 t}{2 \sigma+\varepsilon}\right) d t
$$

Отсюда

$M(r ; \mathscr{G}, f) \leqslant M \exp \left(\sigma+\frac{\varepsilon}{2}\right) \int_{1}^{r} \frac{\alpha(t)}{t} d t \sum_{k=0}^{\infty}(k+1)^{m-1+d} \exp \left(-\int_{0}^{k} \ln \frac{\beta(t / \sigma)}{\beta(2 t /(2 \sigma+\varepsilon))} d t\right)$.

Используя оценки работы [2, с. 133-134], проведенные с учетом условия 4), находим, что ряд в правой части последнего неравенства сходится. Таким образом,

$$
M(r ; \mathscr{G}, f) \leqslant M_{1} \exp \left(\sigma+\frac{\varepsilon}{2}\right) \int_{1}^{r} \frac{\alpha(t)}{t} d t \quad \forall r>0
$$

С учетом

$$
\lim _{r \rightarrow \infty} \int_{1}^{r} \frac{\alpha(t)}{t} d t=+\infty,
$$

получаем, что найдется $r_{\varepsilon}<\infty$ такое, что

$$
\ln M(r ; \mathscr{G}, f) \leqslant(\sigma+\varepsilon) \int_{1}^{r} \frac{\alpha(t)}{t} d t \quad \forall r \geqslant r_{\varepsilon}
$$

В одномерном случае предложения 1 (при $m=1$ ) и 2 (при $m=1, d=0$ ) были ранее получены тем же методом в [3].

Для любой функции

$$
f(z)=\sum_{\|n\|=0}^{\infty} f_{n} z^{n} \in A\left(\mathbb{C}^{m}\right)
$$


положим

$$
\begin{aligned}
& \Omega(F)=\left\{\sigma>0: \exists r_{0}<+\infty \quad \ln M(r ; \mathscr{G}, f) \leqslant \sigma \int_{1}^{r} \frac{\alpha(t)}{t} d t \quad \forall r \geqslant r_{0}\right\}, \\
& \Omega_{1}(F)=\left\{\sigma>0: \exists N<+\infty \quad \ln b_{n} \leqslant-\int_{0}^{\|n\|} \ln \beta\left(\frac{t}{\sigma}\right) d t \quad \forall n \in \mathbb{Z}_{+}^{m},\|n\| \geqslant N\right\}, \\
& \Omega_{2}(F)=\{\sigma>0: \exists d \leqslant+\infty, \quad \exists N<\infty \\
& \left.\ln b_{n} \leqslant d \ln \|n\|-\int_{0}^{\|n\|} \ln \beta\left(\frac{t}{\sigma}\right) d t \quad \forall n \in \mathbb{Z}_{+}^{m},\|n\| \geqslant N,\right\}, \\
& \sigma_{\alpha}(f)=\inf \Omega(f)=\varlimsup_{r \rightarrow \infty}\left(\ln M(r ; \mathscr{G}, f) / \int_{1}^{r} \frac{\alpha(t)}{t} d t\right) \\
& \tau_{\alpha}(f)=\inf \Omega_{1}(f), \quad \lambda_{\alpha}(f)=\inf \Omega_{2}(f) .
\end{aligned}
$$

Из предложений 1,2 непосредственно следует

Tеорема 1. Если $\alpha \in \bar{I}$, mo

$$
\sigma_{\alpha}(f)=\tau_{\alpha}(f)=\lambda_{\alpha}(f) \quad \forall f \in A\left(\mathbb{C}^{m}\right) .
$$

При $m=1$ теорема 1 получена ранее в [2] (для констант $\sigma_{\alpha}(f)$ и $\tau_{\alpha}(f)$ ).

Постараемся придать первому из равенств (3) несколько иную, более симметричную форму, для чего найдем другое представление для $\tau_{\alpha}(f)$. Прежде всего заметим, что если $\alpha \in I$, то обратная к $\alpha$ функция $\beta$ непрерьвно дифференцируема и монотонно возрастает на $[0,+\infty)$, откуда

$$
\beta(x)>1, \quad x \ln \beta(x)>\int_{0}^{x} \ln \beta(t) d t \quad \forall x>0 .
$$

Положим

$$
\varphi(x)=\frac{1}{x} \int_{0}^{x} \ln \beta(t) d t, \quad x \in(0,+\infty), \quad \varphi(0)=0 .
$$

Функция $\varphi$ определена и непрерывна на $[0,+\infty)$, дифференцируема в $(0,+\infty)$. Из (4) следует, что $\varphi^{\prime}(x)>0 \forall x>0$. Кроме того, применение правила Лопиталя дает, что

$$
\lim _{x \rightarrow \infty} \varphi(x)=+\infty
$$

Тогда обратная к $\varphi$ функция $\psi$ определена на $[0,+\infty)$, непрерьвно дифференцируема и монотонно возрастает на $(0,+\infty)$, принимая все значения из $[0,+\infty)$.

Введем еще одну характеристику функции $f$ :

$$
\nu_{\alpha}(f)=\varlimsup_{\|n\| \rightarrow \infty} \frac{\|n\|}{\psi\left(\ln \left(b_{n}\right)^{-1 /\|n\|}\right)},
$$

где, как выше, $b_{n}=\left|f_{n}\right| d_{n}(\mathscr{G}), n \in \mathbb{Z}_{+}^{m}$.

ПРЕДЛОЖЕНИЕ 3. Если $\alpha \in I$, mo $\tau_{\alpha}(f)=\nu_{\alpha}(f) \quad \forall f \in A\left(\mathbb{C}^{m}\right)$. 
ДокАЗАТЕЛЬСтво. 1) Пусть $\nu_{\alpha}(f)<+\infty$, где величина $\nu_{\alpha}(f)$ определена соотношением (5). Тогда $\forall \varepsilon>0 \exists N:\|n\|<\left(\nu_{\alpha}(f)+\varepsilon\right) \psi\left(\ln \left(b_{n}\right)^{-1 /\|n\|}\right) \forall n \in \mathbb{Z}_{+}^{m},\|n\| \geqslant N$. Отсюда при тех же $n$

$$
\ln b_{n}<-\|n\| \varphi\left(\frac{n}{\nu_{\alpha}(f)+\varepsilon}\right)=-\int_{0}^{\|n\|} \ln \beta\left(\frac{t}{\nu_{\alpha}(f)+\varepsilon}\right) d t
$$

Таким образом, $\nu_{\alpha}(f)+\varepsilon \in \Omega_{1}(f) \forall \varepsilon>0$, откуда $\nu_{\alpha}(f) \geqslant \tau_{\alpha}(f)$.

2) Пусть теперь $\tau_{\alpha}(f)<+\infty$. Тогда $\forall \varepsilon>0$ найдется $d<+\infty$ такое, что

$$
\ln b_{n} \leqslant-\int_{0}^{\|n\|} \ln \beta\left(\frac{t}{\tau_{\alpha}(f)+\varepsilon}\right) d t \quad \forall n \in \mathbb{Z}_{+}^{m}, \quad\|n\| \geqslant d .
$$

Последнее неравенство равносильно тому, что

$$
\ln b_{n} \leqslant-\|n\| \varphi\left(\frac{n}{\tau_{\alpha}(f)+\varepsilon}\right)
$$

или, что то же самое, тому, что $\|n\| \leqslant\left(\tau_{\alpha}(f)+\varepsilon\right) \psi\left(\ln \left(b_{n}\right)^{-1 /\|n\|}\right)$. Отсюда $\tau_{\alpha}(f)+\varepsilon \geqslant$ $\|n\| / \psi\left(\ln \left(b_{n}\right)^{-1 /\|n\|}\right) \forall n \in \mathbb{Z}_{+}^{m},\|n\| \geqslant d$. Следовательно, $\tau_{\alpha}(f)+\varepsilon \geqslant \nu_{\alpha}(f) \forall \varepsilon>0$, т.е. $\tau_{\alpha}(f) \geqslant \nu_{\alpha}(f)$.

TeOpema 2. Ecлu $\alpha \in \bar{I}$, mo

$$
\begin{gathered}
\sigma_{\alpha}(f)=\tau_{\alpha}(f)=\nu_{\alpha}(f) \quad \forall f \in A\left(\mathbb{C}^{m}\right) \\
\varlimsup_{r \rightarrow \infty}\left(\ln M(r ; \mathscr{G}, f) / \int_{1}^{r} \frac{\alpha(t)}{t} d t\right)=\varlimsup_{\|n\| \rightarrow \infty} \frac{\|n\|}{\psi\left(\ln \left(b_{n}\right)^{-1 /\|n\|}\right)} .
\end{gathered}
$$

Взяв в качестве $\mathscr{G}$ полидиск $\mathscr{E}_{1}=\left\{z:\left|z_{k}\right|<1, k=1, \ldots, m\right\}$ и положив $M\left(r ; \mathscr{E}_{1}, f\right)=$ $M_{f}(r)$, получим из теоремы 2 с учетом $d_{n}\left(\mathscr{E}_{1}\right)=1 \forall n \in \mathbb{Z}_{+}^{m}$ следующее

СлЕДСТВИЕ. Если $\alpha \in \bar{I} u$

$$
f(z)=\sum_{\|n\|=0}^{\infty} f_{n} z^{n} \in A\left(\mathbb{C}^{m}\right)
$$

mo

$$
\varlimsup_{r \rightarrow \infty}\left(\ln M_{f}(r) / \int_{1}^{r} \frac{\alpha(t)}{t} d t\right)=\varlimsup_{\|n\| \rightarrow \infty} \frac{\|n\|}{\psi\left(\ln \left(f_{n}\right)^{-1 /\|n\|}\right)} .
$$

Если $f$ - любая отличная от полинома функция из $A\left(\mathbb{C}^{m}\right)$, т.е. трансцендентная целая функция, то, как известно (см. [5, гл. II, с. 173]), $\ln M_{f}(r)$ - выпуклая функция от $\ln r$ и

$$
\ln M_{f}(r)=\ln M_{f}(1)+\int_{1}^{r} \frac{u(t)}{t} d t
$$

где функция $u(t)$ монотонно и неограниченно возрастает. Эта функция кусочно-непрерьвна на любом интервале. Более того, промежуток $(1,+\infty)$ можно представить в виде 
объединения $\bigcup_{k=1}^{\infty}\left(x_{k}, x_{k+1}\right), x_{0}=1$, на каждом из которых функция $u$ бесконечно дифференцируема. Ясно, что

$$
\lim _{r \rightarrow \infty}\left(\ln M_{f}(r) / \int_{1}^{r} \frac{u(t)}{t} d t\right)=1 .
$$

Как замечено в [2], всегда можно найти непрерывно дифференцируемую (и даже бесконечно дифференцируемую) в $[1,+\infty)$ функцию $v(x)$ со свойствами 1$)-3)$ такую, что

$$
\varlimsup_{r \rightarrow \infty}\left(\int_{1}^{r} \frac{u(t)}{t} d t / \int_{1}^{r} \frac{v(t)}{t} d t\right)=1
$$

и, следовательно, $\sigma_{v}(f)=1$.

Таким образом, множество $\bar{I}$ является универсальной эффективной шкалой в классе $A\left(\mathbb{C}^{m}\right)$ в том смысле, что для любой $f \in A\left(\mathbb{C}^{m}\right)$ сушествует $\alpha_{0} \in \bar{I}$ такое, что $\sigma_{\alpha_{0}}(f)=1$, и, кроме того, для всех $\alpha \in \bar{I}$ и $f \in A\left(\mathbb{C}^{m}\right)$ справедливо равенство (7).

Обозначим символом $\widehat{I}$ множество всех функций $\alpha(x)$ из $I$, для которых

$$
\varlimsup_{x \rightarrow \infty} \frac{x \alpha^{\prime}(x)}{\alpha^{2}(x)} \int_{1}^{x} \alpha(t) d \ln t \leqslant 2 .
$$

Заметим, что если последнее неравенство имеет место, то, подавно, вьполняется условие 4), так что $\widehat{I} \subset \bar{I}$ (при этом $\widehat{I}$ - собственное подмножество $\bar{I}$ ). Как показанов [3], для любой функции $u(x)$ с перечисленными вьше свойствами найдется функция $v(x)$ из $\widehat{I}$ такая, что

$$
\varlimsup_{r \rightarrow \infty}\left(\int_{1}^{r} u(t) d \ln t / \int_{1}^{r} v(t) d \ln t\right)=1 .
$$

Отсюда следует, что $\widehat{I}$ является универсальной эффективной шкалой для $A\left(\mathbb{C}^{m}\right)$ (при $m=1$ этот результат получен ранее в [3]).

В заключение данного пункта приведем примеры применения формулы (7).

Перед тем, как рассмотреть эти примеры, отметим, что, как и в [2], во всех приведенных вьше (и последуюших) результатах фактически используется лиш асимптотическое (при больших $x$ ) поведение функции $\alpha(x)$ и обратной к ней $\beta(x)$. В частности, можно отказаться от условия $\alpha(1)=0$, заменив во всех интегралах вида

$$
\int_{1}^{r} \frac{\alpha(t)}{t} d t \quad \text { и } \quad \int_{0}^{r} \ln \beta(t) d t
$$

нижние пределы достаточно большими числами.

ПримеР 1. Пусть $\alpha(t)=t^{\rho}, 0<\rho<\infty$. Тогда

$$
\beta(t)=t^{1 / \rho}, \quad \varphi(x)=\frac{1}{\rho} \ln \frac{x}{e}, \quad \psi(x)=\exp (1+\rho x) .
$$

Согласно следствию из теоремы 2

$$
e \rho \varlimsup_{r \rightarrow \infty} \frac{\ln M_{f}(r)}{r^{\rho}}=\varlimsup_{\|n\| \rightarrow \infty}\|n\| \cdot\left|f_{n}\right|^{\rho /\|n\|} \quad \forall f \in A\left(\mathbb{C}^{m}\right)
$$

или, что то же самое,

$$
\left(e \rho \sigma_{f}\right)^{1 / \rho}=\varlimsup_{\|n\| \rightarrow \infty}\|n\|^{1 / \rho}\left|f_{n}\right|^{1 /\|n\|} \quad \forall f \in A\left(\mathbb{C}^{m}\right),
$$

где $\sigma_{f}$ - тип целой функции $f$ порядка $\rho$ (по совокупности переменных). Формула (8) хорошо известна. 
ПримеР 2. Пусть $\alpha(t)=(\ln t)^{\rho}, 0<\rho<\infty$. В этом случае

$$
\beta(t)=\exp \left(t^{1 / \rho}\right), \quad \varphi(x)=\frac{\rho}{\rho+1} x^{1 / \rho}, \quad \psi(x)=\left(\frac{\rho+1}{\rho} x\right)^{\rho} .
$$

По следствию из теоремы 2

$$
(\rho+1)^{\rho+1} \varlimsup_{r \rightarrow \infty} \frac{\ln M_{f}(r)}{(\ln r)^{\rho+1}}=\rho^{\rho} \varlimsup_{\|n\| \rightarrow \infty} \frac{\|n\|^{\rho+1}}{\left(\ln \left(1 /\left|f_{n}\right|\right)\right)^{\rho}} \quad \forall f \in A\left(\mathbb{C}^{m}\right) .
$$

Эта формула также известна (по крайней мере, при $m=1$ ).

ПримеР 3. Пусть, наконец, $\alpha(t)=\exp \left(c(\ln t)^{\rho}\right), 0<c<\infty, 1<\rho<\infty$. Тогда

$$
\beta(x)=\exp \left(\ln \frac{x}{c}\right)^{1 / \rho} .
$$

Как нетрудно подсчитать,

$$
\varphi(x)=c^{-1 / \rho}(\ln x)^{1 / \rho}\left(1-\frac{1}{\rho \ln x}+O\left(\frac{1}{\ln ^{2} x}\right)\right), \quad \psi(t)=\exp \left(c t^{\rho}+1+o(1)\right) .
$$

Согласно следствию из теоремы 2

$$
c \rho e \varlimsup_{r \rightarrow \infty} \frac{\ln M_{f}(r)}{(\ln r)^{1-\rho} \exp \left(c(\ln r)^{\rho}\right)}=\varlimsup_{\|n\| \rightarrow \infty} \frac{\|n\|}{\exp \left(c\|n\|^{-\rho}\left(\ln \left(1 /\left|f_{n}\right|\right)\right)^{\rho}\right)} \quad \forall f \in A\left(\mathbb{C}^{m}\right) .
$$

Заметим, что целые функции $f$, для которых $0<\sigma_{\alpha}(f)<\infty$, имеют в примере 1 ненулевой конечньй, во примере 2 - нулевой (конечньй логариффический) и в примере 3 бесконечный порядок.

Хотя равенства $(6),(7)$ имеют место для любой функции из $A\left(\mathbb{C}^{m}\right)$, при их практическом использовании, особенно в случае целых функций бесконечного порядка (по совокупности переменных), возникают определенные трудности, связанные с необходимостью определения асимптотики (при больших $x$ ) функции $\psi(x)$, обратной к $\varphi(x)$. В связи с этим желательно выразить значение $\nu_{\alpha}(f)$ непосредственно через функции $\alpha(x)$ и $\beta(x)$. Это удается сделать при дополнительных предположениях о функции $\alpha$. Приведем некоторые результаты, полученные в этом направлении, начав с одного вспомогательного утверждения.

ЛЕмма 1 [6]. Пусть функиия $g(x)$ удовлетворяет условиям 1)-3) и условию

$$
\lim _{x \rightarrow+\infty} \frac{x g^{\prime}(x)}{g(x)}=\mu, \quad 0 \leqslant \mu<+\infty .
$$

Тогда

$$
\lim _{t \rightarrow+\infty} \frac{g(k t)}{g(t)}=k^{\mu} \quad \forall k \in(0,+\infty)
$$


Предположим теперь, что функция $\alpha(x)$ из $I$ удовлетворяет условию (9) (при $g=\alpha)$, и пусть $0<\mu<+\infty$. Заметим, что тогда условие 4) выполняется, так что $\alpha \in \bar{I}$. Далее, условие (9) равносильно тому, что

$$
\lim _{x \rightarrow \infty} \frac{x \beta^{\prime}(x)}{\beta(x)}=\frac{1}{\mu}
$$

Применяя лемму 1 к функции $\beta$, получаем

$$
\lim _{k \rightarrow \infty} \frac{\beta(k x)}{\beta(x)}=k^{1 / \mu} \quad \forall k>0 .
$$

Далее,

$$
\varphi(x)=\ln \beta(x)-\frac{1}{x} \int_{0}^{x} \frac{t \beta^{\prime}(t)}{\beta(t)} d t=\ln \beta(x)-\frac{1}{\mu}+o(1) .
$$

Положив $\mu_{1}=\exp (1 / \mu)$, найдем, что $\psi(x)=\alpha\left(\mu_{1}(1+o(1)) \exp x\right)$.

Применяя лемму 1 к функции $\alpha$ и учитывая ее монотонность, имеем

$$
\lim _{x \rightarrow \infty} \frac{\psi(x)}{\alpha(x)}=\mu_{1}^{\mu}=e .
$$

По формуле (5)

$$
e \nu_{\alpha}(f)=\varlimsup_{\|n\| \rightarrow \infty} \frac{\|n\|}{\alpha\left(\left(\left|f_{n}\right| d_{n}(\mathscr{G})\right)^{-1 /\|n\|)}\right.} .
$$

Обозначив через $A$ правую часть последнего равенства, находим

$$
A^{1 / \mu}=\varlimsup_{\|n\| \rightarrow \infty} \beta(\|n\|)\left(b_{n}\right)^{1 /\|n\|} .
$$

Учитьвая теорему 2, получаем

$$
\begin{gathered}
e \sigma_{\alpha}(f)=\varlimsup_{\|n\| \rightarrow \infty} \frac{\|n\|}{\alpha\left(\left(b_{n}\right)^{-1 /\|n\|}\right)} \\
\left(e \sigma_{\alpha}(f)\right)^{1 / \mu}=\varlimsup_{\|n\| \rightarrow \infty} \beta(\|n\|)\left(b_{n}\right)^{1 /\|n\|}, \quad b_{n}=\left|f_{n}\right| d_{n}(\mathscr{G}), \quad n \in \mathbb{Z}_{+}^{m} .
\end{gathered}
$$

Сформулируем полученньй результат.

ТЕорема 3. Если функиия $\alpha$ удовлетворяет условиям 1)-3) и (9), то для любой $f(z) \in A\left(\mathbb{C}^{m}\right)$ справедливы равенства (10) и (11).

Рассмотрим в качестве примера функцию $\alpha(r)=r^{\rho(r)}$, где $\rho(r)$ непрерьвно дифференцируема на $\left[r_{0},+\infty\right)$ и

$$
\lim _{r \rightarrow \infty} l\left(r \ln r \rho^{\prime}(r)+\rho(r)\right)=\mu \in(0,+\infty) .
$$

Тогда условия 1), 2) выполняются (условие 2) - асимптотически), так же как и условие (9). В частности, условие (12) вьполняется, если $\lim _{r \rightarrow \infty} \rho(r)=\rho \in(0,+\infty)$ и 
$\lim _{r \rightarrow \infty} r \ln r \rho^{\prime}(r)=0$, т.е. если $\rho(r)$ - уточненный порядок по Валирону. В этом случае, как нетрудно подсчитать,

$$
\int_{1}^{r} \frac{\alpha(t)}{t} d t \sim \frac{r^{\rho(r)}}{\rho}
$$

и по формуле (11) получаем хорошо известное равенство

$$
(e \rho \tilde{\sigma}(f))^{1 / \rho}=\varlimsup_{n \rightarrow \infty} \beta(\|n\|)\left(\left|f_{n}\right| d_{n}(\mathscr{G})\right)^{1 /\|n\|}
$$

где

$$
\sigma(f)=\varlimsup_{r \rightarrow \infty} r^{-\rho(r)} \ln M(r ; \mathscr{G}, f),
$$

а $\beta(t)$ - функция, обратная к $r^{\rho(r)}$.

Заметим, что теорема 3 "обслуживает", главным образом, целые функции ненулевого конечного порядка. Следуюший результат применяется более широко.

ТЕОРема 4. Пусть функиия $\alpha(x)$ дважды непрерывно дифферениируема на $\left[x_{0},+\infty\right)$ и удовлетворяет условиям 1)-3). Пусть, далее,

$$
\lim _{x \rightarrow \infty} \frac{d \ln \left(x \alpha^{\prime}(x)\right)}{d \ln \alpha(x)}=1 .
$$

Тогда для любой функиии $f \in A\left(\mathbb{C}^{m}\right)$

$$
\nu_{\alpha}(f)=\tau_{\alpha}(f)=\frac{1}{e} \varlimsup_{\|n\| \rightarrow \infty}\left(\beta(\|n\|)\left(\left|f_{n}\right| d_{n}(\mathscr{G})\right)^{1 /\|n\|}\right)^{\beta(\|n\|) /\left(\|n\| \beta^{\prime}(\|n\|)\right)} .
$$

Если, кроме того, функиия $\alpha(x)$ обладает свойством 4), то любая из величин $\nu_{\alpha}(f), \tau_{\alpha}(f)$ в $(14)$, совпадает $c \sigma_{\alpha}(f)$.

Доказательство этой теоремы аналогично доказательству теоремы 2 из [2, с.135-136].

Условиям теоремы 3 удовлетворяют традиционные функции сравнения $\alpha(r)=r^{\rho(r)}$, где $\rho(r) \rightarrow \rho>0$ - уточненньй порядок по Валирону, при дополнительном предположении о том, что функция $\rho(r)$ дважды непрерывно дифференпируема. Как показьвают примеры, приведенные для случая $m=1$ в [2, с. 137-138], этим же условиям удовлетворяют многие функции сравнения, применяемые для описания роста целых функций бесконечного порядка. В то же время ряд функций сравнения, используемых при оценке роста целых функций нулевого порядка, не удовлетворяет условию (13). В качестве примера можно рассмотреть функцию $\alpha(r)=(\ln r)^{\delta}, 0<\delta<\infty$. Соответствуюший $($ при $m=1)$ класс функций $y \in A(\mathbb{C})$, для которых

$$
\varlimsup_{r \rightarrow \infty} \frac{\ln M_{y}(r)}{(\ln r)^{\delta+1}}<\infty
$$

(функции конечного логарифмического порядка), встречается в ряде разделов комплексного анализа, он исследовался Ж. Валироном, А. А. Гольдбергом и другими авторами (см., например, работу [7] и библиографию к ней).

Следующая теорема применима к цельм функциям конечного логарифмического порядка. 
Теорема 5. Пусть функиия $\alpha(x)$ удовлетворяет условиям 1)-3) и, кроме того,

$$
\lim _{x \rightarrow \infty} \frac{\alpha(x)}{x \alpha^{\prime}(x) \ln x}=\gamma, \quad 0<\gamma<+\infty .
$$

Тогда для любой $f \in A\left(\mathbb{C}^{m}\right)$

$$
\left(\nu_{\alpha}(f)\right)^{\gamma}(1+\gamma)=\varlimsup_{\|n\| \rightarrow \infty} \frac{\|n\| \ln \beta(\|n\|)}{\ln \left(1 / b_{n}\right)}, \quad \sigma_{\alpha}(f)=\left[\frac{1}{1+\gamma} \varlimsup_{\|n\| \rightarrow \infty} \frac{\|n\| \ln \beta(\|n\|)}{\ln \left(1 / b_{n}\right)}\right]^{1 / \gamma} .
$$

ДокаЗАТЕЛЬСТво. Прежде всего заметим, что условие (15) влечет условие 4), так что $\alpha \in \bar{I}$ и $\nu_{\alpha}(f)=\tau_{\alpha}(f)=\sigma_{\alpha}(f) \quad \forall f \in A\left(\mathbb{C}^{m}\right)$. Далее, из определения величины $\nu_{\alpha}=\nu_{\alpha}(f)$ следует, что для всякого $\varepsilon>0$ найдется $N<\infty$ такое, что

$$
\|n\|<\left(\nu_{\alpha}+\varepsilon\right) \psi\left(\frac{1}{\|n\|} \ln \frac{1}{b_{n}}\right) \quad \forall n, \quad\|n\|>N .
$$

Отсюда

$$
\varlimsup_{\|n\| \rightarrow \infty} \frac{\|n\| \varphi\left(\|n\| /\left(\nu_{\alpha}+\varepsilon\right)\right)}{\ln \left(1 / b_{n}\right)} \leqslant 1 .
$$

Условие (15) равносильно тому, что

$$
\lim _{x \rightarrow \infty} \frac{\beta^{\prime}(x) x}{\beta(x) \ln \beta(x)}=\gamma
$$

Используя правило Лопиталя, находим

$$
\lim _{x \rightarrow \infty} \frac{\varphi(x)}{\ln \beta(x)}=\lim _{x \rightarrow \infty} \frac{\ln \beta(x)}{\ln \beta(x)+x \beta^{\prime}(x) / \beta(x)}=\frac{1}{1+\gamma} .
$$

Применяя лемму 1 к функции $\ln \beta(x)$, получаем

$$
\lim _{x \rightarrow \infty} \frac{\ln \beta(k x)}{\ln \beta(x)}=k^{\gamma} \quad \forall k>0 .
$$

Из (17) находим

$$
A:=\varlimsup_{\|n\| \rightarrow \infty} \frac{\|n\| \ln \beta(\|n\|)}{\ln \left(1 / b_{n}\right)} \leqslant(1+\gamma)\left(\nu_{\alpha}+\varepsilon\right)^{\gamma}, \quad 0<\varepsilon<\infty .
$$

Следовательно, $A \leqslant(1+\gamma)\left(\nu_{\alpha}\right)^{\gamma}$. С другой стороны, если $\nu_{\alpha}>0$, то для любого $\varepsilon>0$ найдется последовательность мультииндексов $n_{k}$, для которой

$$
\left\|n_{k}\right\| \uparrow \infty, \quad\left\|n_{k}\right\|>\left(\nu_{\alpha}-\varepsilon\right) \psi\left(\frac{1}{\left\|n_{k}\right\|} \ln \frac{1}{b_{n_{k}}}\right), \quad k=1,2, \ldots
$$

Отсюда получим, что для любого $\varepsilon \in\left(0, \nu_{\alpha}\right)$

$$
\varlimsup_{\|n\| \rightarrow \infty} \frac{\|n\| \varphi\left(\|n\| /\left(\nu_{\alpha}-\varepsilon\right)\right)}{\ln \left(1 / b_{n}\right)} \geqslant 1 .
$$


Но тогда

$$
\varlimsup_{\|n\| \rightarrow \infty} \frac{\|n\| \ln \beta(\|n\|)}{\ln \left(1 / b_{n}\right)} \geqslant(1+\gamma)\left(\nu_{\alpha}\right)^{\gamma},
$$

и первое из равенств (16) доказано при $\nu_{\alpha}>0$. Если $\nu_{\alpha}=0$, то из (18) получим

$$
\varlimsup_{\|n\| \rightarrow \infty} \frac{\|n\| \ln \beta(\|n\|)}{\ln \left(1 /\left|b_{n}\right|\right)} \leqslant 0 .
$$

Так как левая часть последнего неравенства неотрицательна, то первое равенство (16) (а, следовательно, и второе) доказано и при $\nu_{\alpha}=0$.

В случае $\gamma=0$ можно также выразить $\nu_{\alpha}(f)$ с помошью функции $\beta$, но соответствующий результат, как мы сейчас убедимся, не является таким законченным, как теорема 5.

ТЕОРема 6. Пусть функиия $\alpha(x)$ удовлетворяет условиям 1)-3), а также условию

$$
\lim _{x \rightarrow+\infty} \frac{\alpha(x)}{x \alpha^{\prime}(x) \ln x}=0 .
$$

Тогда для любой функиии $f \in A\left(\mathbb{C}^{m}\right)$

$$
\begin{aligned}
& \varlimsup_{\|n\| \rightarrow \infty} \frac{\|n\| \ln \beta(\|n\|)}{\ln \left(1 / b_{n}\right)}=1, \text { если } \nu_{\alpha} \in(0,+\infty), \\
& \varlimsup_{\|n\| \rightarrow \infty} \frac{\|n\| \ln \beta(\|n\|)}{\ln \left(1 / b_{n}\right)} \leqslant 1, \quad \text { ecли } \nu_{\alpha}=0 .
\end{aligned}
$$

ДокАЗАТЕЛЬСтво. Пусть $\varepsilon>0$ и $\nu_{\alpha}=\nu_{\alpha}(f) \in[0,+\infty)$. Рассуждая, как при доказательстве теоремы 5 , находим

$$
\varlimsup_{\|n\| \rightarrow \infty} \frac{\|n\| \ln \beta\left(\|n\| /\left(\nu_{\alpha}+\varepsilon\right)\right)}{\ln \left(1 / b_{n}\right)} \leqslant 1 .
$$

Учитьвая, что

$$
\lim _{x \rightarrow \infty} \frac{\ln \beta(k x)}{\ln \beta(x)}=1 \quad \forall k>0,
$$

получим

$$
B_{f}:=\varlimsup_{\|n\| \rightarrow \infty} \frac{\|n\| \ln \beta(\|n\|)}{\ln \left(1 / b_{n}\right)} \leqslant 1 .
$$

Если при этом $\nu_{\alpha}>0$, то для любого $\varepsilon \in\left(0, \nu_{\alpha}\right)$ получаем, как вьше,

$$
\varlimsup_{\|n\| \rightarrow \infty} \frac{\|n\| \ln \beta\left(\|n\| /\left(\nu_{\alpha}-\varepsilon\right)\right)}{\ln \left(1 / b_{n}\right)} \geqslant 1,
$$

откуда $B_{f} \geqslant 1$ и, окончательно, $B_{f}=1$. 
СлЕДСТВИЕ. Пусть функция $\alpha(x)$ удовлетворяет условиям 1)-4) и (19). Тогда для любой функиии $f \in A\left(\mathbb{C}^{m}\right) \quad B_{f}=1$, если $\sigma_{\alpha}(f) \in(0,+\infty), u B_{f} \leqslant 1$, если $\sigma_{\alpha}(f)=0$.

Рассмотрим в качестве примера функцию $\alpha(x)=\exp \left(c(\ln x)^{\mu}\right)$, где $0<c<+\infty$, $0<\mu<+\infty$. Тогда вьполнены условия 1)-4) и (19), причем $\beta(x)=\exp (\ln x / c)^{1 / \mu}$. Положим

$$
\tilde{\sigma}_{f}=\varlimsup_{r \rightarrow \infty} \frac{\ln M(r ; \mathscr{G}, f)}{(\ln r)^{1-\mu} \exp c(\ln r)^{\mu}}, \quad \tilde{\nu}_{f}=\varlimsup_{\|n\| \rightarrow \infty} \frac{\|n\|(\ln \|n\|)^{1 / \mu}}{\ln \left(1 / b_{n}\right)} \quad \forall f \in A\left(\mathbb{C}^{m}\right) .
$$

По теореме 6 , если $\tilde{\sigma}_{f}<+\infty$, то $\tilde{\nu}_{f} \leqslant c^{1 / \mu}$. Если при этом $\tilde{\sigma}_{f} \in(0,+\infty)$, то $\tilde{\nu}_{f}=c^{1 / \mu}$. В частности, если $\mu=1$, то

$$
\begin{gathered}
\alpha(x)=x^{c}, \quad \mu(x)=x^{1 / c} \\
\tilde{\sigma}_{f}=\varlimsup_{r \rightarrow \infty} \frac{\ln M(r ; \mathscr{G}, f)}{r^{c}}, \quad \tilde{\nu}_{f}=\varlimsup_{\|n\| \rightarrow \infty} \frac{\|n\| \ln \|n\|}{\ln \left(1 / b_{n}\right)} .
\end{gathered}
$$

На основании теоремы 6 делаем вьвод, которьй в рамках величин $\tilde{\sigma}_{f}$ и $\tilde{\nu}_{f}$ нельзя улучшить: если $\tilde{\sigma}_{f}=0$, то $\tilde{\nu}_{f} \leqslant \mathrm{c}$, а если $\tilde{\sigma}_{f} \in(0,+\infty)$, то $\tilde{\nu}_{f}=\mathrm{c}$.

2. Целые функции с различным характером роста по переменным. До сих пор мы рассматривали характеристики роста целых функций (типа $M(R ; \mathscr{G}, f)$ ), которые не учитьвали специфику многомерного случая, т.е. возможность различного роста функции $f \in A\left(\mathbb{C}^{m}\right)$ по переменньп $z_{1}, z_{2}, \ldots, z_{m}$. В настоящем пункте приводятся некоторые результаты, в которых такая специфика учитывается.

Положим для любой функции $f(z) \in A\left(\mathbb{C}^{m}\right)$ и для любого $r=\left(r_{1}, \ldots, r_{m}\right) \in \mathbb{R}_{+}^{m}$

$$
M_{f}[r]=\max \left\{|f(z)|:\left|z_{j}\right| \leqslant r_{j}, j=1,2, \ldots, m\right\} .
$$

ПРЕДЛОЖЕНИЕ 4. Пусть непрерывно дифферениируемые на $[1, \infty)$ функиии $\alpha_{j}(x)$ обладают свойствами 1)-3) и $\beta_{j}(x)$ - обратные к ним функиии $(j=1, \ldots, m)$. Пусть, далее, $f \in A\left(\mathbb{C}^{m}\right)$ и существуют $T<\infty, A_{j}<+\infty(1 \leqslant j \leqslant m)$ такие, что

$$
\ln M_{f}[r] \leqslant T+\sum_{j=1}^{m} A_{j} \int_{1}^{r_{j}} \frac{\alpha_{j}(t)}{t} d t \quad \forall r \in \mathbb{R}_{+}^{m} .
$$

Тогда

$$
\ln \left|f_{n}\right| \leqslant T-\sum_{j=1}^{m} \int_{0}^{n_{j}} \ln \beta\left(\frac{t}{A_{j}}\right) d t \quad \forall n \in \mathbb{Z}_{+}^{m} .
$$

ДоКАЗАТЕЛЬСТво. Согласно неравенствам Коши

$$
\left|f_{n}\right| \leqslant M_{f}[r] r^{-n} \quad \forall n \in \mathbb{Z}_{+}^{m}, \quad \forall r \in \mathbb{R}_{+}^{m} .
$$

Отсюда для тех же $n$ и $r$

$$
\ln \left|f_{n}\right| \leqslant T+\sum_{j=1}^{m}\left(A_{j} \int_{1}^{r_{j}} \frac{\alpha_{j}(t)}{t} d t-n_{j} \ln r_{j}\right) .
$$

Положив $r_{j}=\beta_{j}\left(n_{j} / A_{j}\right), j=1, \ldots, m$, получим (после замены $\alpha_{j}(t)=v_{j}$ в каждом из интегралов)

$$
A_{j} \int_{1}^{\beta_{j}\left(n_{j} / A_{j}\right)} \frac{\alpha_{j}(t)}{t} d t-n_{j} \ln \beta_{j}\left(\frac{n_{j}}{A_{j}}\right)=-\int_{0}^{n_{j}} \ln \beta_{j}\left(\frac{t}{A_{j}}\right) d t, \quad j=1,2, \ldots, m .
$$

Следовательно, неравенства (20) установлены. 
ПРЕДЛОЖЕНИЕ 5. Пусть $\alpha \in \bar{I}$,

$$
f(z)=\sum_{\|n\|=0}^{\infty} f_{n} z^{n} \in A\left(\mathbb{C}^{m}\right)
$$

и существуют конечные постоянные $T u A_{j}, j=1, \ldots, m$, такие, что выполняются неравенства (20).

Тогда по любой системе чисел $\left(B_{1}, \ldots, B_{m}\right)$, для которых $B_{j}>A_{j}, j=1, \ldots, m$, найдется конечное число $T_{1}<\infty$ такое, что

$$
\ln M_{f}[r] \leqslant T_{1}+\sum_{j=1}^{m} B_{j} \int_{1}^{r_{j}} \frac{\alpha_{j}(x)}{x} d x \quad \forall r \in \mathbb{R}_{+}^{m} .
$$

ДокАЗАТЕЛЬСТво. Имеем

$$
M_{f}[r] \leqslant \sum_{\|n\|=0}^{\infty}\left|f_{n}\right| r^{n} \leqslant T_{0} \sum_{\|n\|=0}^{\infty} r^{n} \exp \left(-\sum_{j=1}^{m} \int_{0}^{n_{j}} \ln \beta_{j}\left(\frac{t}{A_{j}}\right) d t\right) \quad \forall r \in \mathbb{R}_{+}^{m},
$$

где $T_{0}:=\exp T$. Положим

$$
D_{n_{j}}=\sup _{r_{j}>0}\left\{r_{j}^{n_{j}} \exp \left(-B_{j} \int_{0}^{r_{j}} \frac{\alpha_{j}(t)}{t} d t\right)\right\}
$$

Как отмечалось выше,

$$
D_{n_{j}}=\exp \int_{0}^{n_{j}} \ln \beta\left(\frac{t}{B_{j}}\right) d t, \quad j=1, \ldots, m .
$$

Тогда для всех $r \in \mathbb{R}_{+}^{m}$

$M_{f}[r] \leqslant T_{0} \exp \left(\sum_{j=1}^{m} B_{j} \int_{1}^{r_{j}} \frac{\alpha_{j}(t)}{t} d t\right) \sum_{\|n\|=0}^{\infty} \exp \left(-\sum_{j=1}^{m} \int_{0}^{n_{j}}\left(\ln \beta\left(\frac{t}{A_{j}}\right)-\ln \beta\left(\frac{t}{B_{j}}\right)\right) d t\right)$.

Но, как и вьше (см. [2], [3]), каждьй ряд

$$
S_{j}:=\sum_{n_{j}=0}^{\infty} \exp \left(-\int_{0}^{n_{j}} \ln \frac{\beta\left(t / A_{j}\right)}{\beta\left(t / B_{j}\right)} d t\right), \quad j=1,2, \ldots, m,
$$

сходится. Если $S=\prod_{j=1}^{m} S_{j}$, то

$$
M_{f}[r] \leqslant T_{0} S \exp \left(\sum_{j=1}^{m} B_{j} \int_{1}^{r_{j}} \frac{\alpha_{j}(t)}{t} d t\right) \quad \forall r_{j} \in \mathbb{R}_{+}^{m},
$$

и неравенство (21) доказано. 
Положим $(\alpha)=\left(\alpha_{1}, \ldots, \alpha_{m}\right)$, где $\alpha_{j} \in \bar{I}, 1 \leqslant j \leqslant m,(\beta)=\left(\beta_{1}, \ldots, \beta_{m}\right)$, где $\beta_{j}=\alpha_{j}^{-1}, 1 \leqslant j \leqslant m$. Для любой целой функции

$$
f(z)=\sum_{\|n\|=0}^{\infty} f_{n} z^{n}
$$

введем множества

$$
\begin{aligned}
& E_{f}^{(\alpha)}:=\left\{\gamma \in \mathbb{R}_{+}: \exists T=T(\gamma)<\infty \quad \ln M_{f}[r] \leqslant T+\gamma \sum_{j=1}^{m} \int_{1}^{r_{j}} \frac{\alpha_{j}(t)}{t} d t \quad \forall z \in \mathbb{R}_{+}^{m}\right\}, \\
& E_{f}^{(\beta)}:=\left\{\gamma \in \mathbb{R}_{+}: \exists D=D(\gamma)<\infty \ln \left|f_{n}\right| \leqslant D-\sum_{j=1}^{m} \int_{0}^{n_{j}} \ln \beta_{j}\left(\frac{t}{\gamma}\right) d t \quad \forall n \in \mathbb{Z}_{+}^{m}\right\} .
\end{aligned}
$$

Положим $\sigma(f,\{\alpha\})=\inf E_{f}^{(\alpha)}, \tau(f,\{\beta\})=\inf E_{f}^{(\beta)}$. Из предложений 4,5 непосредственно следует

Теорема 7. Если $\alpha_{j} \in \bar{I}, j=1, \ldots, m$, то для любой иелой в $\mathbb{C}^{m}$ функиии $f$

$$
\sigma(f,\{\alpha\})=\tau(f,\{\beta\}) .
$$

Введем еще два подмножества $\mathbb{R}_{+}^{m}$ :

$$
\begin{aligned}
H_{f}^{(\alpha)}=\left\{A=\left(A_{1}, \ldots, A_{m}\right) \in \mathbb{R}_{+}^{m}: \exists T=T(A)<\infty\right. \\
\left.\ln M_{f}[r] \leqslant T+\sum_{j=1}^{m} A_{j} \int_{1}^{r_{j}} \frac{\alpha_{j}(t)}{t} d t \quad \forall r \in \mathbb{R}_{+}^{m}\right\}, \\
H_{f}^{(\beta)}=\left\{B=\left(B_{1}, \ldots, B_{m}\right) \in \mathbb{R}_{+}^{m}: \exists D=D(B)\right. \\
\left.\ln \left|f_{n}\right| \leqslant D-\sum_{j=1}^{m} \int_{0}^{n_{j}} \ln \beta_{j}\left(\frac{t}{B_{j}}\right) d t \quad \forall n \in \mathbb{Z}_{+}^{m}\right\} .
\end{aligned}
$$

Непосредственно из предложений 4,5 выводим следуюший результат.

Tеорема 8. Eсли $\alpha_{j} \in \bar{I}, j=1, \ldots, m, m o$

$$
\operatorname{int} H_{f}^{(\alpha)}=\operatorname{int} H_{f}^{(\beta)} \quad \forall f \in A\left(\mathbb{C}^{m}\right) .
$$

Заметим, что гиперповерхность $\partial H_{f}^{(\alpha)}$ разбивает $\mathbb{R}_{+}^{m}$ на два множества, одним из которых является int $H_{f}^{(\alpha)}$. Естественно назвать ее зиперповерхностью сопряженных типов по системе $(\alpha)$.

Из теоремы 8 следует, что в ее предположениях гиперповерхность сопряженных типов по системе $(\alpha)$ любой функции $f \in A\left(\mathbb{C}^{m}\right)$ совпадает с гиперповерхностью $\partial H_{f}^{(\beta)}$. 
Tеорема 9. Пусть $\alpha \in \bar{I}$ u $A=\left(A_{1}, \ldots, A_{m}\right) \in \mathbb{R}_{+}^{m}$. Тогда $A \in \partial H_{f}^{(\alpha)}$ (m.e. $\left(A_{1}, \ldots, A_{m}\right)$ - сопряжсенные типы $f$ по системе $\left.(\alpha)\right)$ в том и только том случае, если

$$
\Delta_{f}:=\varlimsup_{\|r\| \rightarrow \infty}\left(\ln M_{f}[r] / \sum_{j=1}^{m} A_{j} \int_{1}^{r_{j}} \frac{\alpha_{j}(t)}{t} d t\right)=1 .
$$

ДокАЗАТЕЛЬСТво. Пусть вьполнено условие (22). Тогда для любого $\varepsilon>0$ найдется $r_{0}$ такое, что

$$
\ln M_{f}[r]<\sum_{j=1}^{m}\left(A_{j}+\varepsilon\right) \int_{1}^{r_{j}} \frac{\alpha_{j}(t)}{t} d t \quad \forall\|r\| \geqslant r_{0}
$$

откуда

$$
\ln M_{f}[r]<C+\sum_{j=1}^{m}\left(A_{j}+\varepsilon\right) \int_{1}^{r_{j}} \frac{\alpha_{j}(t)}{t} d t \quad \forall r \in \mathbb{R}_{+}^{m}
$$

и $\left(A_{1}+\varepsilon, \ldots, A_{m}+\varepsilon\right) \in H_{f}^{(\alpha)} \forall \varepsilon>0$. Следовательно, $A \in \overline{H_{f}^{(\alpha)}}$. Допустим, что $A \in \operatorname{int} H_{f}^{(\alpha)}$. Но тогда найдется $\theta_{0} \in(0,1)$ такое, что $\theta A \in H_{f}^{(\alpha)} \forall \theta \in\left(1,1+\theta_{0}\right)$, откуда

$$
\varlimsup_{\|r\| \rightarrow \infty}\left(\ln M_{f}[r] / \sum_{j=1}^{m} A_{j} \int_{1}^{r_{j}} \frac{\alpha_{j}(t)}{t} d t\right) \leqslant \frac{1}{\theta}<1 .
$$

Следовательно, $A \in \partial H_{f}^{(\alpha)}$.

Обратно, пусть $A \in \partial H_{f}^{(\alpha)}$. Тогда для любого $\varepsilon>0$ найдутся числа $\tilde{A}_{k}, k=1, \ldots, m$, такие, что $\left|\tilde{A}_{k}-A_{k}\right|<\varepsilon$ и $\tilde{A}=\left(\tilde{A}_{k}\right)_{k=1}^{m} \in H_{f}^{(\alpha)}$. Поэтому найдется $C<\infty$ такое, что для всех $r \in \mathbb{R}_{+}^{m}$

$$
\begin{gathered}
\ln M_{f}[r] \leqslant C_{\varepsilon}+\sum_{j=1}^{m} A_{j} \int_{1}^{r_{j}} \frac{\alpha_{j}(t)}{t} d t+\varepsilon \sum_{j=1}^{m} \int_{1}^{r_{j}} \frac{\alpha_{j}(t)}{t} d t \\
\varlimsup_{\|r\| \rightarrow \infty}\left(\ln M_{f}[r] / \sum_{j=1}^{m} A_{j} \int_{1}^{r_{j}} \frac{\alpha_{j}(t)}{t} d t\right) \leqslant 1+\varepsilon \max \left\{\frac{1}{A_{j}}: 1 \leqslant j \leqslant m, A_{j} \neq 0\right\} .
\end{gathered}
$$

Штрих означает суммирование по тем $j \leqslant m$, для которых $A_{j} \neq 0$. Так как $\varepsilon>0$ произвольно мало, то $\Delta_{f} \leqslant 1$. Если $\Delta_{f}<1$, то, очевидно, $A \in \operatorname{int} H_{f}^{(\alpha)}$. Окончательно, $\Delta_{f}=1$.

Аналогичную характеристику гиперповерхности $\partial H_{f}^{(\beta)}$ пока можно дать лишь при дополнительных предположениях относительно функции $\alpha$. Например, если $\alpha \in I$ и выполнено условие (9), то

$$
\begin{aligned}
B= & \left(B_{1}, \ldots, B_{m}\right) \in \partial H_{f}^{(\beta)} \\
& \Longleftrightarrow \varlimsup_{\|n\| \rightarrow \infty}\left(\frac{1}{\|n\|} \ln \left|f_{n}\right|+\sum_{j=1}^{m} \frac{1}{\|n\|} \int_{0}^{n_{j}} \ln \beta_{j}\left(\frac{t}{B_{j}}\right) d t\right)=0 .
\end{aligned}
$$


3. Сравнение с помощью сопряженных функций. Приведенные выше результаты о связи между порядком возрастания модуля целой функции и характером убывания модулей ее тейлоровских коэффициентов такие, как теоремы 1,2 и их многочисленные следствия, имеют тот недостатком, что "не различают" весовые функции, эквивалентные друг другу при $r \rightarrow \infty$. Иначе говоря, если

$$
\lim _{r \rightarrow \infty}\left(\int_{1}^{r} \alpha_{1}(t) d \ln t / \int_{1}^{r} \alpha(t) d \ln t\right)=1, \quad f \in A\left(\mathbb{C}^{m}\right)
$$

то $\sigma_{\alpha_{1}}(f)=\sigma_{\alpha_{2}}(f)$, и как следствие $\nu_{\alpha_{1}}(f)=\nu_{\alpha_{2}}(f)$ (предполагаем, что $\alpha_{j} \in \bar{I}$, $j=1,2)$.

Мы опишем сейчас другой подход к описанию указанной связи, свободный от этого недостатка и основанный на использовании функции, сопряженной с данной в смысле Лежандра-Юнга-Фенхеля (или просто сопряженной).

Пусть $r_{0} \in(0,+\infty)$. Будем говорить, что вещественнозначная функция $H(r)$ удовлетворяет условиям $(\mathrm{A})$, если $H(r)$ определена и ограничена снизу на любом промежутке $\left[r_{0}, M\right], M<+\infty$, и если $\lim _{r \rightarrow \infty}(H(r) / r)=+\infty$.

Если $H(r)$ удовлетворяет условиям $(\mathrm{A})$, то на $[0,+\infty)$ определена сопряженная к ней функция $H^{*}(x)=\sup _{r \geqslant r_{0}}[x r-H(r)]$.

Как известно (см., например, [8, гл. III, § 12$]), H^{*}(r)$ не убьвает на $[0,+\infty)$, т.е. $H^{*}(x) \geqslant H^{*}(0)=-\inf _{r \geqslant r_{0}} H(r) \quad \forall x \in[0,+\infty)$, причем

$$
\lim _{x \rightarrow \infty} \frac{H^{*}(x)}{x}=+\infty
$$

Поэтому на $[0,+\infty)$ определена сопряженная к $H^{*}$ функция

$$
H^{* *}(x)=\sup _{t \geqslant 0}\left\{x t-H^{*}(t)\right\}
$$

Для нее $H(r) \geqslant H^{* *}\left(r_{0}\right) \forall r \geqslant r_{0}$, и равенство $H(r)=H^{* *}(r), r \geqslant r_{0}$, имеет место тогда и только тогда, когда $H(r)$ выпукла на $\left[r_{0},+\infty\right)$. Так, в частности, будет, если для $H(r)$ выполнены условия $(\mathrm{A}), H(r)$ непрерывно дифференцируема на $\left[r_{0},+\infty\right), \mathrm{a}$ $H^{\prime}(r)$ монотонно возрастает. В этом случае, если $H_{1}(x)$ - обратная к $H^{\prime}(x)$ функция, то $H^{*}(x)=x H_{1}(x)-H\left(H_{1}(x)\right), x \geqslant x_{1}$.

Пусть теперь $\mathscr{G}$ - ограниченная полная кратнокруговая область в $\mathbb{C}^{m}$ с центром в начале координат и

$$
f(z)=\sum_{\|n\|=0}^{\infty} f_{n} z^{n} \in A\left(\mathbb{C}^{m}\right) .
$$

Положим для любого $r>0$

$$
\mu_{f}(r, \mathscr{G})=\sup _{\|n\| \geqslant 0}\left\{\left|f_{n}\right| d_{n}(\mathscr{G}) r^{\|n\|}\right\}=\sup _{\|n\| \geqslant 0}\left\{b_{n} r^{\|n\|}\right\} .
$$

Пусть, кроме того, $r_{1}=\exp r_{0}$. 
Теорема 10. Если Н удовлетворяет условиям (А), то

$$
\sup _{n \in \mathbb{Z}_{+}^{m}}\left\{\ln b_{n}+H^{*}(\|n\|)\right\}=\sup _{r \geqslant r_{1}}\left\{\ln \mu_{f}(r, \mathscr{G})-H(\ln r)\right\} \quad \forall f \in A\left(\mathbb{C}^{m}\right) .
$$

ДокАЗАТЕЛЬСТво. Пусть сначала правая часть равенства (23) конечна и равна $d$. Тогда $\ln \mu_{f}(r, \mathscr{G}) \leqslant H(\ln r)+d \forall r \geqslant r_{1}$. Отсюда

$$
\begin{gathered}
\ln b_{n} \leqslant d+H(\ln r)-\|n\| \ln r, \quad \ln b_{n} \leqslant d-\sup _{x \geqslant x_{0}}\{\|n\| x-H(x)\}=d-H^{*}(n) \\
\forall n \in \mathbb{Z}_{+}^{m}, \quad \forall r \geqslant r_{1} .
\end{gathered}
$$

Пусть теперь левая часть равенства (23) конечна и равна $D$. Тогда $\ln b_{n} \leqslant D-H^{*}(\|n\|)$ $\forall n \in \mathbb{Z}_{+}^{m}$. Отсюда

$$
\ln b_{n} r^{\|n\|} \leqslant D+\sup _{x \geqslant 0}\left\{x \ln r-H^{*}(r)\right\}=D+H^{* *}(\ln r) \leqslant D+H(\ln r) \quad \forall r \leqslant r_{1} .
$$

Следовательно, $\mu_{f}(r, \mathscr{G}) \leqslant D+H(\ln r) \forall r \geqslant r_{1}$.

Приведем теперь оценку для $M(r ; \mathscr{G}, f)$, хорошо известную при $m=1$ :

$$
\begin{gathered}
M(r ; \mathscr{G}, f) \leqslant \sum_{\|n\|=0}^{\infty} b_{n} r^{\|n\|} \leqslant \sum_{\|n\|=0}^{\infty} b_{n}(\theta r)^{\|n\|} \theta^{-\|n\|} \leqslant T(\theta) \mu_{f}(\theta r, \mathscr{G}) \\
\forall r>0, \quad \forall \theta \in(1,+\infty) .
\end{gathered}
$$

Введем следуюшие характеристики целой функции $f$ :

$$
\begin{aligned}
& \delta(f ; \mathscr{G}, H)=\inf \left\{c>0: \varlimsup_{r \rightarrow \infty}(\ln M(r ; \mathscr{G}, f)-H(\ln r+c))<+\infty\right\}, \\
& \gamma(f ; \mathscr{G}, H)=\varlimsup_{\|n\| \rightarrow \infty}\left(\ln \left(b_{n}\right)^{1 /\|n\|}+\frac{H^{*}(\|n\|)}{\|n\|}\right) .
\end{aligned}
$$

ТЕОРема 11. Если функиия $H(x)$ удовлетворяет условиям (А) и не убъвает на $\left[x_{0},+\infty\right), m o$

$$
\delta(f ; \mathscr{G}, H)=\gamma(f ; \mathscr{G}, H) \quad \forall f(z) \in A\left(\mathbb{C}^{m}\right) .
$$

ДокАЗАТЕЛЬСТво. Положим $\delta=\delta(f ; \mathscr{G}, H), \gamma=\gamma(f ; \mathscr{G}, H)$.

1) Пусть сначала $\gamma<+\infty$. Тогда для любого $\varepsilon>0$ найдется $D<+\infty$ такое, что

$$
\ln b_{n}+H^{*}(\|n\|)<\left(\gamma+\frac{\varepsilon}{2}\right)\|n\|+D \quad \forall n \in \mathbb{Z}_{+}^{m} .
$$

Выберем число $\theta>1$ так, чтобы $2 \ln \theta<\varepsilon$. Для всех $r>0$ имеем

$$
\begin{gathered}
M(r ; \mathscr{G}, f) \leqslant \sum_{\|n\|=0}^{\infty} b_{n} r^{\|n\|} \leqslant T(\theta) \sup _{\|n\| \geqslant 0}\left\{b_{n}(\theta r)^{\|n\|}\right\}, \\
\ln M(r ; \mathscr{G}, f) \leqslant T_{1}(\theta)+\sup _{\|n\| \geqslant 0}\left\{-H^{*}(\|n\|)+D+\left(\gamma+\frac{\varepsilon}{2}\right)\|n\|+\|n\| \ln r+\|n\| \ln \theta\right\} \\
\leqslant T_{2}(\theta)+\sup _{x \geqslant 0}\left\{x(\gamma+\varepsilon+\ln r)-H^{*}(x)\right\} \leqslant T_{2}(\theta)+H(\ln r+\gamma+\varepsilon) .
\end{gathered}
$$


Отсюда

$$
\lim _{r \rightarrow \infty}(\ln M(r ; \mathscr{G}, f)-H(\ln r+\gamma+\varepsilon))<\infty \quad \forall \varepsilon>0,
$$

и, следовательно, $\gamma+\varepsilon \geqslant \delta \forall \varepsilon>0$, т.е. $\gamma \geqslant \delta$.

2) Пусть теперь $\delta<+\infty$. Тогда для любых $n \in \mathbb{Z}_{+}^{m}$ и $\varepsilon>0$ найдется $Q<\infty$ такое, что

$$
\begin{aligned}
\ln b_{n} & \leqslant \ln M(r ; \mathscr{G}, f)-\|n\| \ln r \leqslant Q+H(\ln r+\delta+\varepsilon)-\|n\| \ln r \\
& =Q+\|n\|(\delta+\varepsilon)-(\|n\|(\ln r+\delta+\varepsilon)-H(\ln r+\delta+\varepsilon)) .
\end{aligned}
$$

Отсюда

$$
\ln b_{n} \leqslant Q+\|n\|(\delta+\varepsilon)-\sup _{t \geqslant 0}\{\|n\| t-H(t)\}=Q+\|n\|(\delta+\varepsilon)-H^{*}(\|n\|) \quad \forall n \in \mathbb{Z}_{+}^{m} .
$$

Следовательно, $\ln \left(b_{n}\right)^{1 /\|n\|}+H^{*}(\|n\|) /\|n\| \leqslant Q /\|n\|+\delta+\varepsilon$, откуда $\gamma \leqslant \delta+\varepsilon \forall \varepsilon>0$, т.е. $\gamma \leqslant \delta$. Окончательно, $\gamma=\delta$.

Рассмотрим некоторые примеры. Пусть $f \in A\left(\mathbb{C}^{m}\right), \mathscr{G}=\mathscr{E}_{1}=\left\{z=\left(z_{1}, \ldots, z_{n}\right)\right.$ : $\left.\left|z_{k}\right| \leqslant 1, k=1, \ldots, n\right\}$. Тогда $d_{n}\left(\mathscr{E}_{1}\right)=1 \forall n \in \mathbb{Z}_{*}^{m}$. Положим $M_{f}(r)=M\left(r ; \mathscr{E}_{1}, f\right)$. Тогда, как известно, $\ln M_{f}(r)$ - выпуклая функция от $\ln r$ :

$$
\ln M_{f}(r)=s+\int_{1}^{r} \frac{\nu(t)}{t} d t
$$

При этом, если $f$ - трансцендентная целая функция (т.е. $f$ - не полином), то функция $\nu$ монотонно и неограниченно возрастает. Положим

$$
H_{0}(x)=s+\int_{1}^{e^{x}} \frac{\nu(t)}{t} d t=\ln M_{f}\left(e^{x}\right)
$$

Тогда $H_{0}(x)$ - выпуклая функция от $x$ на $(0, \infty)$, монотонно и неограниченно возрастающая. Для всякого $c \in(-\infty,+\infty)$ положим

$$
\delta_{c}:=\varlimsup_{r \rightarrow \infty}\left(\ln M_{f}(r)-H_{0}(\ln r+c)\right) \leqslant 0 .
$$

Легко проверить, что $\delta_{c} \leqslant 0$ при $c \geqslant 0, \delta_{0}=0$ и $\delta_{c}=+\infty \forall c>0$. Таким образом, $\delta\left(f ; \mathscr{E}_{1}, H_{0}\right)=0$ и по теореме 11

$$
\varlimsup_{\|n\| \rightarrow \infty}\left(\ln \left|f_{n}\right|^{1 /\|n\|}+\frac{H_{0}^{*}(\|n\|)}{\|n\|}\right)=0 .
$$

При этом $H_{0}(x)$ - вьпуклая функция, определенная на $[0,+\infty)$. Последнее равенство, пожалуй, наиболее точно характеризует порядок убывания тейлоровских коэффициентов целой функции с заданньм максимумом модуля (в определенном смысле это равенство неулучшаемо).

В качестве примера рассмотрим функцию $H(x)=g(x)+\nu x$, где функция $g(x)$ непрерьвна, дифференцируема и положительна на $\left[x_{0},+\infty\right)$, причем $\lim _{x \rightarrow+\infty} H^{\prime}(x)=+\infty$ 
(и, следовательно, $\left.\lim _{x \rightarrow \infty}(H(x) / x)=+\infty\right)$. В данном случае $H^{*}(t)=g^{*}(t-\nu)$ и по теореме 10

$$
\begin{aligned}
& \varlimsup_{\|n\| \rightarrow \infty}\left(\ln \left(b_{n}\right)^{1 /\|n\|}+\frac{g^{*}(\|n\|-\nu)}{\|n\|}\right) \\
& \quad=\inf \left\{c>0: \varlimsup_{r \rightarrow \infty}(\ln M(r ; \mathscr{G}, f)-g(\ln r+c)-\nu \ln r)<+\infty\right\} \quad \forall f \in A\left(\mathbb{C}^{m}\right) .
\end{aligned}
$$

Если, в частности, $g(x)=x^{2}$, то, как легко подсчитать,

$$
\varlimsup_{r \rightarrow \infty} \frac{\ln M(r ; \mathscr{G}, f)-(\ln r)^{2}}{\ln r}=\varlimsup_{\|n\| \rightarrow \infty}\left(\ln \left|b_{n}\right|^{1 /\|n\|}+\frac{\|n\|}{4}\right) \quad \forall f \in A\left(\mathbb{C}^{m}\right) .
$$

Таким образом, теорема 11 позволяет охарактеризовать порядок убывания тейлоровских коэффициентов целых функций, рост максимума модуля которых имеет двучленную (и даже более сложную) асимптотику.

В заключение рассмотрим уже знакомый случай, когда

$$
H(r)=\int_{1}^{\exp r} \alpha(t) d \ln t
$$

Пусть $I_{1}-$ класс всех непрерывных, монотонно возрастающих на $[1,+\infty)$ функций, удовлетворяюших условиям 1), 3) из п. 1 (как и вьше, условие 1) может быть опущено). Очевидно, что $I$ - собственное подмножество $I_{1}$. Пусть $\alpha \in I_{1}$. Тогда $H(r)$ - вьпуклая функция от $r$, причем

$$
H^{\prime}(r)=\alpha\left(e^{r}\right), \quad H_{1}(r)=\ln \beta(r), \quad H^{*}(r)=\int_{0}^{r} \ln \beta(t) d t .
$$

Пусть $f \in A\left(\mathbb{C}^{m}\right)$ и $\gamma=\gamma(f ; \mathscr{G}, H), \delta=\delta(f ; \mathscr{G}, H)$. Имеем

$$
\gamma=\ln \varlimsup_{\|n\| \rightarrow \infty}\left(b_{n}\right)^{1 /\|n\|_{1}} \beta_{n}
$$

где

$$
\beta_{n}:=\exp \left(\frac{1}{\|n\|}\right) \int_{0}^{\|n\|} \ln \beta(t) d t .
$$

Что же касается величины $\delta$, то в данной ситуации ее можно представить в несколько иной форме. Прежде всего, заметим, что если $\delta<+\infty$, то для любого $\varepsilon>0$ найдется $d<+\infty$ такое, что

$$
\ln M(r ; \mathscr{G}, f) \leqslant d+\int_{1}^{r \exp (\delta+\varepsilon)} \alpha(t) d \ln t \quad \forall r \geqslant r_{0} .
$$

Тогда при $r \geqslant r_{\varepsilon}$

$$
\ln M(r ; \mathscr{G}, f) \leqslant \int_{1}^{b(r)} \alpha(t) d \ln t, \quad b(r)=r \exp (\delta+2 \varepsilon) .
$$


Положим $\zeta(r)=\int_{1}^{r} \alpha(t) d \ln t$. Так как $\zeta(r)$ непрерьвно и монотонно возрастает на $[1,+\infty)$, то обратная к ней функция $\zeta^{-1}(r)$ определена на $[0,+\infty)$. При этом для всех $r \geqslant r_{1}$ вьполнено $\zeta^{-1}(\ln M(r ; \mathscr{G}, f)) \leqslant b(r)$. Положим

$$
\eta:=\varlimsup_{r \rightarrow \infty} \frac{\zeta^{-1}(\ln M(r ; \mathscr{G}, f))}{r} .
$$

Имеем $\eta \leqslant \exp (\delta+2 \varepsilon) \forall \varepsilon>0$, откуда $\eta \leqslant \exp \delta$.

Пусть теперь $\eta<+\infty$. Тогда $\ln M(r ; \mathscr{G}, f) \leqslant \zeta(r(\eta+\varepsilon)) \forall \varepsilon>0, \forall r>\widetilde{r}_{\varepsilon}$, и потому

$$
\varlimsup_{r \rightarrow \infty}(\ln M(r ; \mathscr{G}, f)-\zeta(r(\eta+\varepsilon)))<+\infty \quad \forall \varepsilon>0 .
$$

Отсюда $\eta+\varepsilon \geqslant \exp \delta \forall \varepsilon>0$, т.е. $\eta \geqslant \exp \delta$. В итоге $\eta=\exp \delta$, и, учитьвая равенство (24), приходим к следующему результату.

ТЕОрема 12. Если $\alpha \in I_{1}$, то для любой функиии $f \in A\left(\mathbb{C}^{m}\right)$

$$
\varlimsup_{r \rightarrow \infty} \frac{\zeta^{-1}(\ln M(r ; \mathscr{G}, f))}{r}=\varlimsup_{\|n\| \rightarrow \infty}\left(b_{n}\right)^{1 /\|n\|} \exp \left(\frac{1}{\|n\|}\right) \int_{0}^{\|n\|} \ln \beta(t) d t,
$$

где $\mathscr{G}$ - любая ограниченная полная кратнокруговая область с иентром в начале координат, $b_{n}=\left|f_{n}\right| d_{n}(\mathscr{G}), \beta(t)-$ функиия, обратная $\kappa \alpha(r), a \zeta^{-1}-\kappa \int_{1}^{r} \alpha(t) d \ln t$.

\section{СПИСОК ЦИТИРОВАННОЙ ЛИТЕРАТУРЫ}

[1] Левин Б. Я. Распределение корней целых функций. М.: Гостехтеоретиздат, 1956.

[2] Осколков В. А. О некоторых вопросах теории целых функций // Матем. сб. 1993. Т. 184. №1. С. 129-148.

[3] Осколков В.А. Свойства функций, заданных значениями их линейных функционалов. Дисс. ‥ к.ф̆-м. н. М.: МГУ, 1995.

[4] Ронкин Л.И. Элементы теории аналитических функций многих переменных. Киев: Наукова думка, 1977.

[5] Ронкин Л.И.Введение в теорию целых функций многих переменных. М.: Наука, 1971.

[6] Сенета Е. Правильно меняющиеся функции. М.: Наука, 1985.

[7] Братищев А. В., Коробейник Ю. Ф. О некоторых характеристиках роста субгармонических функций // Матем. сб. 1978. Т. 106. №1. С. 44-65.

[8] Рокафеллар Р. Выпуклый анализ. М.: Мир, 1973.

Ростовский государственный университет

E-mail : kor@rsu.rnd.su 